\title{
The HIV/HBV co-infected patient: Time for proactive
}

\section{management}

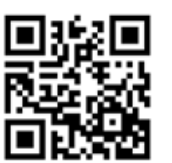

Worldwide an estimated 240 million people are chronically infected with chronic hepatitis B virus $(\mathrm{CHB})$, of whom an estimated 3.4 million are coinfected with HIV. ${ }^{[1]}$ Chronic liver disease has emerged as an important cause of morbidity in the HIV-infected population. It is, after opportunistic infections, the second most common cause of death among some populations of HIV-infected patients on antiretroviral therapy (ART). ${ }^{[2]}$ In resource-limited settings (RLSs), infection with HIV and hepatitis B virus (HBV) is associated with poorer outcomes than HBV mono-infection. Co-infection is associated with higher rates of HBV persistence after acute infection, and among individuals with $\mathrm{CHB}$, higher HBV DNA levels, a higher prevalence of $\mathrm{HBV}$ e antigenaemia, and an increased risk of progression to fibrosis, cirrhosis and possibly hepatocellular carcinoma (HCC). ${ }^{[2,3]}$ Identifying patients who are chronically infected and providing specific management improves outcomes. ${ }^{[4]}$ However, screening for active HBV infection before starting ART is not currently part of HIV guidelines in many countries in sub-Saharan Africa (SSA).

\section{Epidemiology}

Much of SSA has been considered to have high endemicity of CHB $(\geq 8 \%)$. In Zimbabwe prevalence rates as high as $25 \%$ in an antenatal cohort, and rates of $13 \%$ in Malawi, $10.6 \%$ in Botswana and $17.3 \%$ in HIV-infected adults in Tanzania, have been reported. However, more recent data suggest that the prevalence in SSA may be lower than previously thought, probably owing to wide variations in prevalence across countries; for example, in South Africa (SA) the prevalence of hepatitis B surface antigen (HBsAg) as a marker of active HBV infection ranges from $3.5 \%$ to $23 \%{ }^{[5-7]}$ Nevertheless, the morbidity coupled with the overall high endemicity of $\mathrm{CHB}$ is reason to screen for this infection and initiate optimal management for those found to be infected.

\section{Goals of treatment and drug therapy}

The primary goal of treating $\mathrm{CHB}$ is to reduce the risk of progressive liver disease, cirrhosis and HCC by suppressing HBV replication. The management cascade starts with diagnosing CHB by testing for HBsAg. This can be done by rapid point-of-care assays. If HBsAg is persistently detectable in the blood for $>6$ months, the patient is diagnosed with $\mathrm{CHB}$. In patients positive for $\mathrm{HBs} A g$, additional testing may include hepatitis $\mathrm{B} e$ antigen (HBeAg), antibody to hepatitis B e antigen (anti-HBe), and HBV DNA qualitatively and quantitatively. Intermediate goals and markers of treatment success are suppression of HBV replication as measured by the $\mathrm{HBV}$ viral load, $\mathrm{HBeAg} /$ anti-HBe seroconversion (in $\mathrm{HBeAg}$-positive patients), HBsAg/anti-HBs seroconversion (preceded by a reduction in HBsAg titre), alanine aminotransferase normalisation and improvement of liver histological features. Viral eradication is not currently a goal of therapy, as none of the available therapeutic agents clear the highly stable covalently closed circular DNA (cccDNA) from hepatocytes.

A number of agents are available for the treatment of $\mathrm{CHB}$, of which some also have HIV activity. A reverse transcriptase step in the HBV lifecycle, whereby pregenomic HBV RNA is transcribed to viral DNA, is the explanation for this. Screening for HIV in the HBV-infected patient before starting ART is therefore critical to avoid inadequate HIV therapy.

Tenofovir is among the most potent and effective agents for treating CHB. Lamivudine also has activity against HBV, but is less potent and has a high rate of failure and resistance development when used as a single HBV-active agent. ${ }^{[8]}$ Entecavir also has potent activity against HBV but minimal activity against HIV. It is generally the treatment of choice when renal impairment makes tenofovir use problematic. Although available in SA, it is prohibitively expensive. Other HBV-specific agents have been developed (telbivudine, adefovir), but they are expensive, have a poorer resistance profile and are not widely available. Daily interferon (IFN) or weekly pegylated IFN therapy to treat and potentially clear $\mathrm{HBsAg}$ (but not cccDNA) is a treatment option in the mono-infected patient. However, it achieves lower rates of HBeAg seroconversion among co-infected patients, especially in the HBV genotypes common in SA, and is poorly tolerated.

Risks and benefits of treating $\mathrm{CHB}$ should be assessed before initiating therapy. Among co-infected patients who are eligible for ART, ART should always include HBV-active agents - ideally tenofovir. There is probably a subset of $\mathrm{CHB}$ patients with a low risk of progressive liver disease. Patients who are HBeAg-negative, have low HBV viral loads and lack evidence of liver fibrosis may not benefit from treatment. It is notable that the use of transaminases for monitoring liver disease, which assists in assessing treatment need among HIV-uninfected individuals, may have limited value in HIV infection because of lower transaminase values despite ongoing necroinflammatory disease and fibrosis. Any HBV-infected patient with cirrhosis or stigmata of chronic liver disease should receive antiviral therapy. All HIV/HBV co-infected patients with any evidence of liver disease (elevated transaminase levels, elevated HBV DNA titres, necroinflammation and fibrosis on biopsy) should be started on HBV-active therapy irrespective of CD4 count. ${ }^{[9]}$ The most recent SA HIV treatment guidelines (December 2014) include known HBV infection as an indication to start ART, irrespective of CD4 count.

\section{Addressing other risks}

Lifestyle modifications such as reducing or curtailing alcohol intake will minimise the additional risk of cirrhosis. Advice about avoiding over-thecounter herbal remedies and traditional medications is important. The metabolic syndrome is associated with both HIV and the development of non-alcoholic steatohepatitis (NASH). NASH may lead to cirrhosis and HCC. Risk factors for NASH and advanced fibrosis in the HBV mono-infected patient are age, diabetes, obesity, exposure to stavudine, and the metabolic syndrome. Vaccination against hepatitis A is indicated in those who are non-immune. The cirrhotic patient requires additional intervention and surveillance, e.g. gastroscopy.

\section{Monitoring, managing and stopping HBV therapy}

After initiation of agents active against $\mathrm{HBV}$ in the HIV/HBV co-infected patient, HBV DNA levels decline far more gradually than HIV RNA levels. If HBV DNA monitoring is available, it is reasonable to perform a test after 12 months of therapy. If there is failure to achieve HBV suppression by 12 months despite good adherence suggested by undetectable HIV RNA, entecavir added to tenofovir-containing therapy may result in better HBV control. Costeffective and practical methods for monitoring HBV DNA that will be valuable in RLSs, such as point-of-care HBV viral load tests and testing of dried blood spots, are under development.

Following initiation of ART, transaminase levels commonly increase transiently among co-infected patients. This is usually attributed to druginduced hepatotoxicity, but other causes should be considered (Table 1). The reported incidence of ART-related hepatotoxicity is between $5 \%$ and $10 \%$ in the HIV-infected population, with a higher rate in co-infected patients. ${ }^{[9]}$ Some agents, particularly nevirapine, are more likely to cause hepatotoxicity than others and should be avoided in patients with liver disease. Lopinavir/ritonavir given at higher doses in the presence of rifampicin-containing antituberculosis medication may induce mild to moderate hepatotoxicity. Most episodes are brief, lasting around 2 - 4 


\section{EDITORIAL}

Table 1. Causes of liver enzyme elevation during ART

Drug effects - ART related and non-ART related

Poor adherence

Inadvertent stopping of anti-HBV agents, e.g. changing from first- to second-line ART

Infection with another agent, e.g. TB, HAV, HEV

Hepatic steatosis (associated with chronic ART use and the metabolic syndrome)

Toxins, e.g. alcohol and herbal medicines

Worsening of underlying liver disease, e.g. autoimmune liver disease

Emergence of $\mathrm{HBV}$ resistance

$\mathrm{TB}=$ tuberculosis; $\mathrm{HAV}=$ hepatitis $\mathrm{A}$ virus; $\mathrm{HEV}=$ hepatitis $\mathrm{E}$ virus.

weeks, and resolve without intervention. Discontinuation of ART may be another cause of liver inflammation, either as part of a regimen change or through a lapse in adherence. Stopping HBV-active ART can lead to a rapid rise in HBV replication, leading to symptoms that can range from subclinical to fulminant hepatitis, and may mimic acute HBV infection. This syndrome generally occurs several weeks to months after stopping therapy, but may occasionally occur within days. Drug resistance rarely emerges in patients on potent therapy such as tenofovir. ${ }^{[10]}$

\section{Screening for HCC}

Routine $\mathrm{CHB}$ management in resource-rich settings includes regular screening for HCC. American Association for the Study of Liver Diseases (AASLD) guidelines recommend an ultrasound scan of the liver every 6 - 12 months for those at risk of developing HCC. ${ }^{[11]}$ Alpha-fetoprotein screening at 6-monthly intervals lacks sensitivity for early HCC. ${ }^{[12]}$ Studies on optimal screening methods, intervals and evaluations of costeffectiveness in HIV/HBV co-infected populations in RLSs are needed.

\section{Occult HBV infection}

Occult HBV infection is partially controlled HBV infection, usually associated with low levels of HBV DNA $\left(<10^{4} \mathrm{IU} / \mathrm{mL}\right)$ and an HBsAgnegative serological profile. Antibodies to $\mathrm{HBV}$ core (anti-HBc) may or may not be present. Estimates of the prevalence of occult $\mathrm{HBV}$ infection among HIV-infected patients in Africa vary between $2 \%$ and $85 \%$, depending on population, study design and extent of immune deficiency. ${ }^{[13]}$ Recovery of CD4 counts, as occurs with ART, can lead to the resolution of occult HBV infection. The overall clinical importance of occult HBV infection is unclear, and there are currently no guidelines that recommend screening for this condition.

\section{HBV vaccination}

$\mathrm{HBV}$ vaccination is routinely provided in many high-income countries for adults in high-risk groups. Cost, and perhaps a lack of appreciation of the frequency of adult HBV transmission, have discouraged implementation of similar programmes in many RLSs, including SA. Although there are few data on acute HBV infection among adults in most of SSA, infection can and does occur, especially among higher-risk populations. One study from SA reported an annual incidence of newly acquired HBV infection of $2.5 \%$ among young women at high risk for HIV. ${ }^{[14]}$ Only approximately a quarter of these women had evidence of prior exposure (anti-HBc antibodies), leaving the majority of the population at risk of infection. Given this incidence of HBV, the practice of vaccinating nonimmune HIV-infected individuals would seem reasonable.

Because individuals with CD4 counts $<200$ cells/ $\mu \mathrm{L}$ have a poor immune response to vaccination, vaccinating adults with higher CD4 counts is likely to have greater public health impact. Data are needed to better understand the cost-effectiveness of HBV vaccination in HIV-infected adults in RLSs.
The commencement of routine infant HBV vaccination, currently at 6 weeks of age, should be shifted to closer to the time of birth in order to prevent mother-to-child transmission (MTCT) of HBV.

\section{Conclusion}

With the roll-out of ART in RLSs, the emphasis of care is shifting to maintaining quality of life and managing chronic conditions. Given the high prevalence of HBV infection in SSA and the risk of HIV/HBV co-infected patients developing liver complications, the management of HBV/HIV should be prioritised. In SSA efforts need to be focused on screening for chronic viral hepatitis, early institution of effective antiviral therapy, vaccinating those who are most at risk of infection, and the prevention of HBV MTCT.

\section{I Andersson \\ W Preiser}

Division of Medical Virology, Department of Pathology, Faculty of Medicine and Health Sciences, Stellenbosch University, Tygerberg, Cape Town, South Africa, and National Health Laboratory Service Tygerberg, Cape Town

\section{C van Rensburg}

Division of Gastroenterology and Hepatology, Department of Internal Medicine, Faculty of Medicine and Health Sciences, Stellenbosch University, Tygerberg, Cape Town, South Africa

\section{J Taljaard}

Division of Infectious Diseases, Department of Internal Medicine, Faculty of Medicine and Health Sciences, Stellenbosch University, Tygerberg, Cape Town, South Africa

\section{J Hoffmann}

Johns Hopkins University, Baltimore, MD, USA, and Aurum Institute, Johannesburg, South Africa

\section{Corresponding author: M I Andersson (andersson_m@sun.ac.za)}

1. Puoti M, Airoldi M, Bruno R, et al. Hepatitis B virus co-infection in human immunodeficiency virusinfected subjects. AIDS Rev 2002;4(1):27-35 PMID 11998781

2. Thio CL, Seaberg EC, Skolasky R, et al. HIV-1, hepatitis B virus, and risk of liver-related mortality in the Multicenter Cohort Study (MACS). Lancet 2002;360(9349):1921-1926. [http://dx.doi.org/10.1016/ S0140-6736(02)11913-1]

3. Salmon-Ceron D, Rosenthal E, Lewden C, et al. Emerging role of hepatocellular carcinoma amon liver-related causes of deaths in HIV-infected patients: The French national Mortalité 2005 study. Hepatol 2009;50(4):736-745. [http://dx.doi.org/10.1016/j.jhep.2008.11.018]

4. Chen C-J, Yang H-I. Natural history of chronic hepatitis B REVEALed. J Gastroenterol Hepatol 2011;26(4):628-338. [http://dx.doi.org/10.1111/j.1440-1746.2011.06695.x]

5. Ott JJ, Stevens GA, Groeger J, Wiersma ST. Global epidemiology of hepatitis B virus infection: New estimates of age-specific HBsAg seroprevalence and endemicity. Vaccine 2012;30(12):2212-2219. estimates of age-specific HBsAg seroprevalence
[http://dx.doi.org/10.1016/j.vaccine.2011.12.116]

6. Andersson MI, Maponga TG, Ijaz S, et al. The epidemiology of hepatitis B virus infection in HIV-infected and HIV-uninfected pregnant women in the Western Cape, South Africa. Vaccine HIV-infected and HIV-uninfected pregnant women in the Western Cape

2013;31(47):5579-5584. [http://dx.doi.org/10.1016/j.vaccine.2013.08.028]
7. Lukhwareni A, Burnett RJ, Selabe SG, Mzileni MO, Mphahlele MJ. Increased detection of HBV DNA in HBsAgpositive and HBsAg-negative South African HIV/AIDS patients enrolling for highly active antiretroviral therapy at a tertiary hospital. J Med Virol 2009;81(3):406-412. [http://dx.doi.org/10.1002/jmv.21418]

8. Benhamou $Y$, Bochet $M$, Thibault $V$, et al. Long-term incidence of hepatitis $B$ virus resistance to lamivudine in human immunodeficiency virus-infected patients. Hepatology 1999;30(5):1302-1306 [http://dx.doi.org/10.1002/hep.510300525]

9. Soriano V, Puoti M, Bonacini M, et al. Care of patients with chronic hepatitis B and HIV co-infection Recommendations from an HIV-HBV International Panel. AIDS 2005;19(3):221-240. [http://dx.doi. org/10.1097/01.aids.0000163948.62176.e7]

10. Audsley J, Arrifin N, Yuen LK, et al. Prolonged use of tenofovir in HIV/hepatitis B virus (HBV)coinfected individuals does not lead to HBV polymerase mutations and is associated with persistence of lamivudine HBV polymerase mutations. HIV Med 2009;10(4):229-235. [http://dx.doi.org/10.1111/ of lamivudine HBV polym

11. Lok ASF, McMahon BJ. Chronic hepatitis B: Update 2009. Hepatology 2009;50(3):661-662. [http:// dx.doi.org/10.1002/hep.23190]

12. Aghoram R, Cai P, Dickinson JA. Alpha-foetoprotein and/or liver ultrasonography for screening of hepatocellular carcinoma in patients with chronic hepatitis B. Cochrane Database Syst Re 2012, Issue 9. Art. No.: CD002799. [http://dx.doi.org/10.1002/14651858.CD002799.pub2]

13. Cohen Stuart JW, Velema M, Schuurman R, Boucher CA, Hoepelman AI. Occult hepatitis $B$ in persons infected with HIV is associated with low CD4 counts and resolves during antiretroviral therapy. J Med Virol 2009;81(3):441-445. [http://dx.doi.org/10.1002/jmv.21422]

14. Baxter C, Yende-Zuma N, Tshabalala P, Abdool Karim Q, Abdool Karim SS. Safety of coitally administered tenofovir $1 \%$ gel, a vaginal microbicide, in chronic hepatitis B virus carriers: Results from the CAPRISA 004 trial. Antiviral Res 2013;99(3):405-408. [http://dx.doi.org/10.1016/j.antiviral.2013.06.019]

S Afr Med J 2015;105(4):281-282. DOI:10.7196/SAMJ.8907 